\title{
DNA editing in mouse embryos prevents disease
}

\section{Destroying faulty DNA in mitochondria could be alternative to 'three-parent embryo'.}

\section{Sara Reardon}

23 April 2015

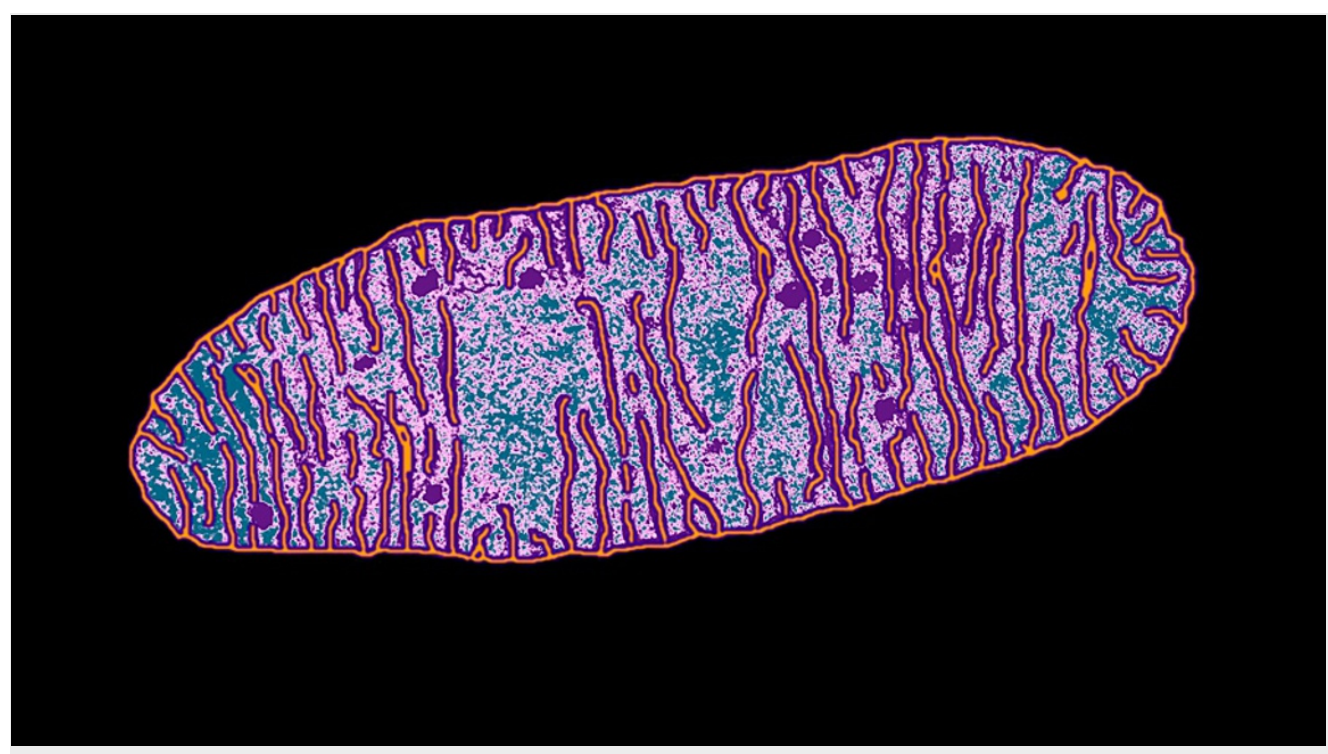

Dr. Don Fawcett/Getty

A typical egg carries hudnreds of thousands of mitochondria, such as the one shown here.

Mothers may one day be able to prevent their children from inheriting mitochondrial defects. Therapies that modify diseased eggs are inching closer to the clinic, but researchers are still hotly debating the safety and ethics of the most promising techniques. These involve combining the nucleus of the mother's egg with mitochondria from a healthy woman to create a 'three-parent embryo'.

In the 23 April issue of Cell ${ }^{1}$, one team proposes an alternative: neutralizing the faulty mitochondria. Some researchers say that the approach could help enable the ethically questionable practice of engineering human embryos to have modifications that would be passed on to future generations. (The first instance of such engineering was published in Protein \& Cell ${ }^{2}$ and reported by Nature's news team on 22 April.)

About 1 in 5,000 people worldwide has a disorder caused by faulty mitochondria, the organelles that supply the cell with energy. In others, the faulty organelles worsen diseases that arise by other means, such as some cancers. Roughly $60-95 \%$ of the mitochondria in a cell must be faulty for disease to develop ${ }^{3}$. In most cases, however, just a small fraction of the hundreds of the thousands of mitochondria in an egg carry flaws, says Alejandro Ocampo, a molecular biologist at the Salk Institute for Biological Studies in La Jolla, California, and an author of the Cell study.

Ocampo and Juan Carlos Izpisua Belmonte, a developmental biologist at Salk, realized that reducing the amount of mutant mitochondrial DNA in an egg or fertilized embryo could reduce the chance of disease developing. They did this by injecting the cells with a segment of RNA that was designed to produce a DNA-cutting enzyme known as an endonuclease. The endonuclease would then seek out mitochondria with a specific mutation and destroy their DNA by snipping it.

Ocampo and Izpisua Belmonte ran their experiment on mouse eggs and one-cell embryos that contained mitochondria with DNA from two different mouse strains. They injected the cells with RNA designed to create endonucleases that knock out DNA from just one strain, and implanted the embryos into surrogate mothers. Tests revealed that around $60 \%$ of the mitochondrial DNA from the targeted strain had been destroyed — and the resulting baby mice were healthy.

In a second experiment, the researchers fused mouse eggs with mitochondria from people with mitochondrial diseases; in these cells, the customized RNA eliminated $20-50 \%$ of damaged mitochondrial DNA. 


\section{Early results}

The technique is still far from being available to parents. Editing human germ cells and embryos is illegal in many countries and is widely considered unethical ${ }^{4,5}$. In recent weeks, several research groups have called for a moratorium on such work until guidelines can be developed to delineate legally and ethically acceptable research or clinical applications. Izpisua Belmonte and Ocampo are waiting for approval from ethics boards before applying their technique to human cells.

Even then, the researchers would still need to prove that their approach does not damage the embryo, says molecular biologist Michal Minczuk of the University of Cambridge, UK. Destroying a large proportion of an embryo's mitochondrial DNA could make it difficult for the embryo to implant in the uterus, he says.

And molecular geneticist Eric Shoubridge of McGill University in Montreal, Canada, says that it could be difficult to engineer RNA for individual patients, because there are so many mutations that cause mitochondrial disease.

The approach could eliminate the need for 'three-parent embryo' techniques, which involve moving nuclei or mitochondria between eggs. This can damage the cells, and the overall concept has given many scientists and lawmakers pause. By contrast, injecting customized RNA "is something that any IVF clinic can do with their eyes closed", Izpisua Belmonte says.

Marcy Darnovsky, director of the Center for Genetics and Society in Berkeley, California, says that the approach would also avoid many of the concerns posed by the concept of three-parent embryos, which her group opposes. But she remains sceptical that these methods will make it into the clinic any time soon, as creating a healthy child is not the same as curing a disease - and therefore may be outweighed by the risks. And in the strictest sense, she adds, mitochondrial editing still creates modifications in the germline.

"It is a bit of a slippery slope — if you start allowing any editing tool, you open a Pandora's box of the possibility to edit anything," says Shoubridge. "Whether we want to go down that road is an ethical question. I'm not sure we do."

Scientific hurdles might also remain. Douglas Turnbull, a neurologist at Newcastle University, UK, who pioneered the transfer of nuclei into healthy donor eggs, says that the mouse work is elegant and exciting. But he notes that eggs from women with high numbers of faulty mitochondria may not survive the knockout process, because so little of the organelles' DNA would remain.

Nevertheless, Ocampo and Izpisua Belmonte say that they are in the process of acquiring discarded human eggs and embryos from a fertility clinic, and waiting for approval from an ethics board. They plan to develop a line of stem cells from these modified cells, but say that they will not implant embryos into mothers or allow them to grow.

Nature | doi:10.1038/nature.2015.17379

\section{References}

1. Reddy, P. et al. Cell http://dx.doi.org/10.1016/j.cell.2015.03.051 (2015).

2. Liang, P. et al. Protein Cell http://dx.doi.org/10.1007/s13238-015-0153-5 (2015).

3. Russell, O. \& Turnbull, D. Exp. Cell Res. 325, 38-43 (2014).

4. Lanphier, E. et al. Nature 519, 410-411 (2015).

5. Baltimore, D. et al. Science 348, 36-38 (2015). 